Conference Presentation

\title{
Two cases reports comparing histopathological features of canine transmissible venereal tumor after treatment with decimal potencies of Viscum Album and Vincristin
}

\author{
Leoni Villano Bonamin', Gisela Novaes Fontao Lefebvre ${ }^{2}$, Thayna Neves Cardoso', Marcus \\ Reif ${ }^{3}$
}

${ }^{1}$ Research Center, Paulista University, Sao Paulo, Brazil.

${ }^{2}$ Veterinary Doctor, Brazil.

3IKF-Berlin, Germany.

Email: leonibonamin@gmail.com

\begin{abstract}
The aim of the study was to evaluate the histopathological features of two canine transmissible venereal tumor (TVT) cases: one treated with Viscum album (Q) protocol in serial decimal potencies and the other with Vincristin conventional protocol (treated control). Tumor infiltrating leukocytes pattern and apoptosis were analyzed quantitatively, using a computer image analysis system, twice: before the beginning of treatment and after six weeks. Both female dogs were sujected to weekly clinical evaluation for three months, focusing on tumor remission and eventual adverse effects manifestations (local or systemic). In both the cases, animals had a round cells tumor growth, with weak intercellular stroma connection, typical of a TVT mass. The lysozyme positivity in immunohistochemistry analysis, associated with typical morphology confirmed the diagnosis. Ten fields were randomly photographed by a digital photomicrography system NIKON Eclipse 2 - Coolpix. Each captured image was analyzed quantitatively by the Metamorph® software, considering the number of leukocyte infiltrated and mitosis per field. Caspase 3 positivity index was also evaluated. In Vincristin treated dog, the number of leukocytes infiltrating tumor was bigger than that found in Viscum album treated dog, even before the beginning of the treatment, indicating an initial conditions compatible to a more favorable prognosis, however, the number of leukocytes increased in the Viscum album treated dog during the observation period (Kruskal Wallis, $\mathrm{p}<0.05$ ). The animal treated with Vincristine has no statistical significant variation in the number of infiltrating mononuclear cells during the treatment, but presented 10 times more mitotic figures (Kruskal Wallis, $\mathrm{p}<0.001$ ) in the second biopsy, compared to the other stages of treatment. There was a statistical significant increase in the rate of positivity for Caspase 3 between biopsies taken before and after treatment with Viscum album (ANOVA, p=0.01), but not after Vincristin treatment. Even though, the tumor remission could be achieved only in Vincristin treated dog, with an abrupt reduction of mitosis number and tumor mass in the last 20 days of treatment. In contrast, a secondary infection of the tumor led to clinical worsening of TVT in Viscum album treated dog, which could explain the increase in the local leukocytes number. The increase in Caspase 3

OPEN ACCESS

Cite as: Bonamin LV, Lefebvre GNF, Cardoso TN, Reif M. Two Cases Report Comparing Histopathological Features of Canine TVT after Treatment with Decimal Potencies of Viscum Album or Vincristin. Proceedings of the XXIX GIRI Meeting; 2015 June 3 - 5; Verona (Italy). Int J High Dilution Res. 2015; 14(2): 55-56
\end{abstract}


Proceedings of the XXIX GIRI Meeting; 2015 June 3 - 5; Verona (Italy). International Journal of High Dilution Research 2015; 14(2): 55-56.

Available online at www.highdilution.org

positivity after treatment shows the Viscum album anti-neoplastic mechanism of action, but in this case it was not enough to reach a complete tumor remission.

Keywords: Transmissible venereal tumor, Viscum album, Vincristin, dog, clinical report.

(C) International Journal of High Dilution Research.

Not for commercial purposes.

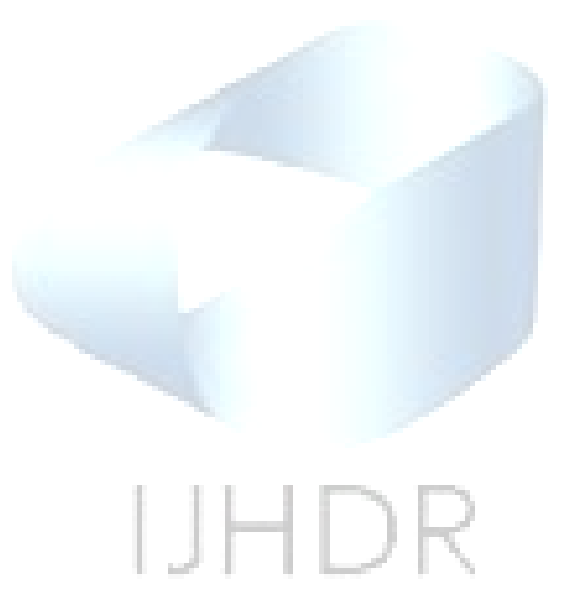

OPEN ACCESS

Cite as: Bonamin LV, Lefebvre GNF, Cardoso TN, Reif M. Two Cases Report Comparing Histopathological Features of Canine TVT after Treatment with Decimal Potencies of Viscum Album or Vincristin. Proceedings of the XXIX GIRI Meeting; 2015 June 3 - 5; Verona (Italy). Int J High Dilution Res. 2015; 14(2): 55-56 